ticularly testing problem for the relativistic beaming theories popular among VLBI observers, as the high apparent brightness temperatures (up to $10^{15} \mathrm{~K}$ ) require high- $\gamma$ jets very close to the line of sight, and consequently cause statistical embarrassments in hiding the numerous jets not pointing at us. It was therefore particularly interesting to hear of two classes - those whose variations correlate well with variations above 1 $\mathrm{GHz}$, and the rest whose low-frequency variations may have a different physical cause - and that BL Lac is the archetype of the former class. Unfortunately very few objects can as yet be classified in this way.

Sherwood and colleagues (Max Planck Institute) reported a very high detection rate of optically selected quasars at $\lambda \sim 1$ $\mathrm{mm}$; the fluxes lie on an extrapolation of the optical spectra and well above the extrapolated radio spectra. Infra-red observations were reviewed by Rieke (University of Arizona) and exploited by Lilly (Royal Observatory, Edinburgh) and Puschell, Owen and Laing (NRAO) to obtain spectrophotometric redshift estimates for very distant radio galaxies.

The fact that radio-quiet quasars have stronger $\mathrm{Fe}$ II lines than radio-loud quasars now appears well established (Oosterbrock, University of California, Santa Cruz; B Wills, University of Texas), but the weakness of the iron lines may be associated only with the steep radio spectrum sources with extended structure while the flat-spectrum core-dominated sources have iron lines more like the radioquiet quasars.

The observations of large-scale ( $\gtrless 1 \mathrm{kpc}$ ) jets strongly suggest that their speeds are a few tenths of a c or less, while the prevalence of fast variations and superluminal velocities (always outward) makes it difficult for VLBI observers to abandon the idea of ultrarelativistic jets on a small scale. Apart from statistical difficulties in losing the many quasars whose jets do not point at us, there are theoretical difficulties in slowing down jets without quietly losing very large amounts of energy. The only case where we are sure of the speed is the galactic source SS433, in which VLA observations incontrovertibly show a pair of precessing jets (thanks to a period of 164 days instead of megayears). Hjellming (NRAO) presented a comprehensive analysis leading to a distance of $5.5 \mathrm{kpc}$ and an ejection speed of $0.26 \mathrm{c}$, the latter confirming earlier optical work. How far should one take the analogy with larger specimens? Only one or two of the 200 or so maps shown resembled SS433 (specifically, one shown by Gower, University of Victoria), though several more showed possible evidence of precession. If a jet originates near a black hole it is expected to be fast, but not with a lorentzian factor $\geq 5$ unless the jet consists of electrons and positrons rather than electron-ion plasma (Rees, University of Cambridge). Thorne (Caltech), in a magnificent exposition of the ways of precessing a black hole and of

\title{
Exploiting geomagnetic data
}

\section{from S.R.C. Malin and D.R. Barraclough}

IT costs about $£ 20,000$ per year to run a modern, efficient geomagnetic observatory. (It costs very little less to run an obsolete, inefficient observatory, but that is another story.) Whilst annual mean values are not the only output from observatories, they are some of the most valuable, particularly for studies of the main geomagnetic field and its secular variation, which provide the only information we have on the detailed dynamics of the Earth's core. Accurate estimates of the secular variation require a continuous series of annual mean values extending over a number of years. In view of the high cost of acquiring these data, it is desirable that the effort put into analysing them does full justice to their information content. This has seldom been possible in the past.

Over the past few years, the development of inverse theory (see the review by Parker A. Rev. Earth planet. Sci. 5; 35, 1977) has provided the means of deriving not only optimum models from given data sets but also reliable estimates of their uncertainty. Because of the physical complexity of the Earth's magnetic field and the mathematical complications of inverse theory, the application of this method to geomagnetic field modelling has been long delayed. In a recent paper, Whaler and Gubbins (Geophys. $J l R$. astr. Soc. 65; 645, 1981) have gone some way towards rectifying this.

Most previous attempts to model the geomagnetic field have used the classical methods of spherical harmonic analysis and have paid scant attention to errors. Whaler and Gubbins examine the validity of such procedures in the light of the recent developments in inverse theory and enquire whether a better description of the field might not be obtained from a somewhat different analysis of the data. Much of the work is involved with the mathematics of casting the problem in a form suitable for treatment by linear inverse theory. As an example of the use of the method, the authors apply it to a set of data based on observatory annual mean values and compare the resulting spherical harmonic coefficients with

extracting energy from it, showed that if one feeds it a balanced diet, including magnetic field, it will inevitably produce a Poynting flux and very probably electronpositron pairs.

Baldwin (University of Cambridge) discussed the evolutionary tracks of individual sources in the size-luminosity plane. On the evolution of the source population and the double quasar, Maarten Schmidt, in a conciliatory mood, allowed that as many as 2 per cent of quasars could be local objects, while Barnothy appeared at last in his rightful those derived by the classical method. Such a data set is very small compared with those used in modelling the main geomagnetic field but is similar to those used in some studies of the secular variation. The authors concede that computational costs would prevent the extension of the method to large data sets. Although inverse theory can, in principle, give realistic estimates of the uncertainties of the resulting model, several technical problems have yet to be solved before such estimates can be obtained in the geomagnetic case. The main conclusion of this part of the paper, that the coefficients derived by inverse theory have little advantage over those derived by classical means, may seem trivial. However, this is far from being an obvious result and it is valuable to have it set on a firm mathematical basis.

The derivation of spherical harmonic coefficients is only part of the problem. For studies of core dynamics we require optimum estimates of the values of the field components at the surface of the core, together with their uncertainties. Whaler and Gubbins examine this part of the problem in terms of the spherical harmonic coefficients and the distribution of the data from which they are derived, again using the methods of inverse theory. While they reach no very firm conclusions, their formulation of the problem and their investigation of various smoothing procedures break valuable new ground and lay the foundations for future work.

Application of inverse theory to the modelling of the main geomagnetic field and its secular variation should thus help to exploit more fully the costly observations which are the basis of our knowledge of the field. Whaler and Gubbins have made a beginning; it remains for field modellers in general to assimilate their results and apply them, where possible, to the production of future models.

S.R.C. Malin and D.R. Barraclough are in the Geomagnetism Unit, Institute of Geological Sciences, Edinburgh.

role as a member of the astronomical establishment.

Finally, in the most intriguing talk of the symposium, Rudnick (University of Minnesota) exhibited transparencies to demonstrate that hotspots and other bright features are not truly symmetric, but rather interleave when their positions are reflected through the optical nuclei. Rudnick concluded that the nuclear engine ejects on one side at a time; but there are stranger implications than that, which will shed much new darkenss on theoretical models if the facts are confirmed. 